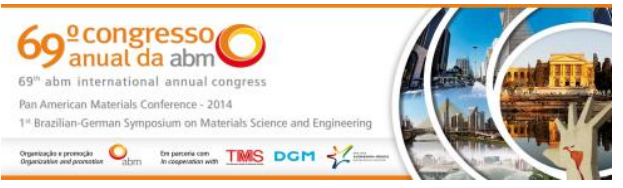

Tema: Produtos metálicos não-ferrosos

\title{
INFLUÊNCIA DO FLUXO GASOSO NA REDUÇÃO DE ÓXIDO DE COBRE SOBRE PLASMA DE HIDROGÊNIO*
}

\author{
Luciana Pirone de Oliveira ${ }^{1}$ \\ Estéfano Aparecido Vieira ${ }^{2}$ \\ Simão Vervloet Ramos ${ }^{3}$
}

\section{Resumo}

O cobre é encontrado na natureza na forma de óxidos ou sulfetos e sua obtenção na forma metálica é usualmente alcançada através da pirometalurgia e eletrólise. Em ambos os processos há, além do gasto com energia, a geração de grande quantidade de passivos ambientais. Neste contexto a redução via plasma de hidrogênio se apresenta como uma alternativa promissora, pois valores significativos de carga metálica podem ser obtidos em temperaturas inferiores aos processos usuais e tendo como resíduo apenas vapor de água. Neste trabalho procurou-se investigar a influência do fluxo de hidrogênio na eficiência da redução do $\mathrm{Cu}_{2} \mathrm{O}$ por plasma de hidrogênio em baixa temperatura. Os experimentos foram realizados em reator de plasma pulsado CC nas vazões de 100, 200, 300 e 400 $\mathrm{cm}^{3} / \mathrm{min}$, com temperatura constante de $300^{\circ} \mathrm{C}$ por $1 \mathrm{~h}$ e pressão de 4Torr. A eficiência da redução foi determinada através de um balanço de massa e as análises dos produtos da redução foram feitas por difração de raios-x (DRX) e microscopia eletrônica de varredura (MEV). Os resultados parciais ratificam a redução a plasma como nova rota para a produção de cobre metálico e demonstram que as variações do fluxo de hidrogênio têm pouca influência na eficiência da redução.

Palavras-chave: Redução; Óxido de cobre; Plasma de hidrogênio.

\section{INFLUENCE OF GASEOUS FLOW IN COPPER OXIDE REDUCTION ON HYDROGEN PLASMA}

\begin{abstract}
Copper is found in nature in the form of oxides or sulphides, and obtaining the metallic form is usually achieved by pyrometallurgical and electrolysis. In both cases there is, besides spending on energy, the generation of large amount of environmental liabilities. In this context the reduction by hydrogen plasma is presented as a promising alternative because significant amounts of metal loading can be obtained at temperatures lower than usual processes as waste and taking only water vapor. In this study we sought to investigate the influence of hydrogen flow on the efficiency of the reduction of $\mathrm{Cu}_{2} \mathrm{O}$ by hydrogen plasma at a low temperature. The experiments were performed in DC pulsed plasma reactor in flow rates of $100,200,300$ and $400 \mathrm{~cm}^{3} / \mathrm{min}$ a constant temperature of $300^{\circ} \mathrm{C}$ for $1 \mathrm{~h}$ and pressure 4Torr. The efficiency of the reduction was determined by a mass balance and analysis of the reduction products were made by $\mathrm{x}$ - ray diffraction $(\mathrm{XRD})$ and scanning electron microscopy (MEV). The results confirm the reduction in plasma as a new route for the production of metallic copper and demonstrate that the changes in hydrogen flow have little influence on the efficiency of the reduction.

Keywords: Reduction; Copper oxide; Hydrogen plasma.

1 Graduanda em Engenharia Metalúrgica. Instituto Federal de educação, ciência e tecnologia do Espírito Santo, IFES, Campus Vitória, Espírito Santo, Brasil.

2 Professor Doutor, Programa de Pós Graduação em Engenharia Metalúrgica e de Materiais (Propemm), IFES, Campus Vitória, Espírito Santo, Brasil.

3 Mestrando, Propemm, IFES, Campus Vitória, Espírito Santo, Brasil.
\end{abstract}

* Contribuição técnica ao 69ำ Congresso Anual da ABM - Internacional e ao 14을 ENEMET Encontro Nacional de Estudantes de Engenharia Metalúrgica, de Materiais e de Minas, 21 a 25 de julho de 2014, São Paulo, SP, Brasil. 


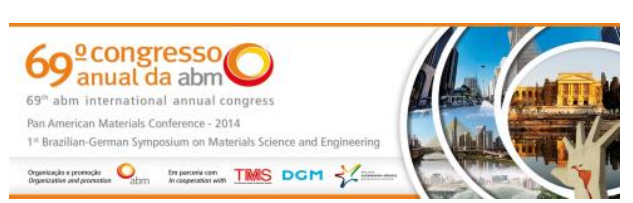

\section{INTRODUÇÃO}

O cobre é o segundo metal não ferroso mais utilizado no mundo, depois apenas do alumínio [1]. Sua grande utilização se deve às suas peculiaridades como boa condutividade elétrica e térmica, resistência à corrosão, ductilidade, maleabilidade e comportamento não magnético [2].

O cobre em estado puro, denominado cobre nativo, raramente é encontrado na natureza. Normalmente está associado a outros elementos químicos em várias formas estruturais, proporções estequiométricas e combinações químicas, formando diversos minerais [3]. Por ter grande afinidade pelo enxofre, seus minérios são geralmente sulfetados, mas existe uma boa parte de oxidados (óxidos, silicatos, carbonatos, sulfatos [4].

Existem dois processos básicos de produção de cobre: o processo pirometalúrgico, mais utilizado para os minérios sulfetados, e o processo hidrometalúrgico de concentração de minérios seguido de eletrólise. Este último sendo mais apropriado para a extração de cobre de minérios oxidados de baixo teor. Como característica comum ambos apresentam, além do grande consumo de energia, a geração de passivos ambientais [5].

Segundo a Caraíba Metais S/A [6], empresa localizada na Bahia, que produz cobre pelo processo pirometalúrgico, para cada tonelada de cobre produzido são gastos $1442,4 \times 10^{3} \mathrm{kWh}$ de energia elétrica e $152,2 \times 10^{6} \mathrm{~m}^{3}$ de gás natural. Além disso, são produzidos $3,6 \times 10^{3} \mathrm{~m}^{3}$ de efluentes e 0,2 toneladas de resíduos sólidos. Outro fator importante é o consumo de água que chega a 10,8 x $10^{6} \mathrm{~m}^{3}$ de água/ $\mathrm{t}$ de $\mathrm{Cu}$ produzido. Estes valores ilustram o impacto ambiental decorrente da produção de cobre pelos processos atuais e justifica a pesquisa de novas rotas mais "limpas" para a obtenção de Cu metálico.

No presente trabalho objetivou-se estudar a obtenção do Cu metálico a partir do seu óxido mais estável, na forma de pó de alta pureza, mediante uma nova vertente dos processos de redução direta: ou seja, a redução assistida por plasma em baixa temperatura. Este processo se justifica em duas situações: i) quando se deseja produzir o metal com alto grau de pureza; ii) quando se objetiva reduzir de forma significativa a geração de resíduos. Além disso, possui a vantagem de promover a redução sob temperaturas mais baixas quando comparado com os processos convencionais.

A redução de óxidos metálicos empregando-se o hidrogênio é amplamente utilizada na produção de catalisadores e dispositivos eletrônicos. No caso do óxido de cobre a redução se daria segundo a reação (1). Porém, a cinética da reação só se torna viável para elevados valores de temperatura [7]:

$$
\mathrm{Cu}_{2} \mathrm{O}_{(\mathrm{s})}+\mathrm{H}_{2} \rightarrow \mathrm{Cu}_{(\mathrm{s})}+\mathrm{H}_{2} \mathrm{O}_{(\mathrm{g})}
$$

O plasma de hidrogênio apresenta como possibilidade a produção de espécies de alto poder redutor, derivadas da molécula de $\mathrm{H}_{2}{ }^{(7)}$. Várias reações ocorrem com as espécies gasosas presentes no plasma. A tabela a seguir resume estes fenômenos.

\footnotetext{
* Contribuição técnica ao 69ำ Congresso Anual da ABM - Internacional e ao 14을 ENEMET Encontro Nacional de Estudantes de Engenharia Metalúrgica, de Materiais e de Minas, 21 a 25 de julho de 2014, São Paulo, SP, Brasil.
} 


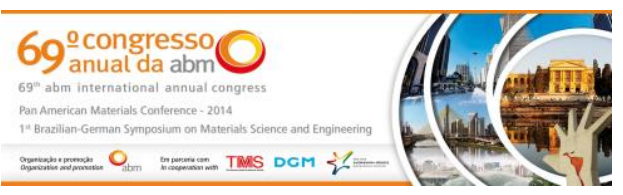

concomitantemente pelas espécies excitadas de hidrogênio e pelo hidrogênio molecular.

\section{MATERIAIS E MÉTODOS}

\subsection{Aquisições dos materiais}

O óxido de cobre (I) na forma de pó utilizado nos experimentos foi adquirido da empresa JB Química, São Paulo - SP. Possui teor mínimo de 97,00\% de $\mathrm{Cu}_{2} \mathrm{O}$ e $87,00 \%$ de $\mathrm{Cu}$. Granulometria de 325 mesh. O gás hidrogênio utilizado para as reduções foi fornecido pela White Martins, com pureza de 99,999\%.

Para as reduções, foram utilizados cadinhos de aço inoxidável austenítico AISI 316L.

\subsection{Redução a Plasma}

Os experimentos de redução foram realizados usando um reator a plasma pulsado da marca THOR NP SDS, pertencente ao laboratório do grupo de engenharia de superfícies enges do instituto federal espírito santo- IFES.

Basicamente, o equipamento é constituído por:

- saídas para medidas de pressão, temperatura, tempo da tensão ligada e tensão máxima.

- câmara cilíndrica de aço inoxidável diâmetro 500 mm e altura 750 mm.

- entradas para o gás de redução;

- bomba de vácuo e outros acessórios necessários à redução dos pós.

\subsection{Redução}

Para determinação do grau de redução nas diferentes condições, as amostras foram colocadas em 5 cadinhos de aço inoxidável $316 \mathrm{l}$, de $30 \mathrm{~mm}$ de diâmetro por $3 \mathrm{~mm}$ de altura, cada uma na quantidade de $200 \mathrm{mg}$. Os cadinhos contendo as amostras foram pesados em uma balança semi-analítica com precisão de 0,001g do laboratório de redução o IFES. Após a redução foram novamente pesados para a determinação do grau de redução por análise gravimétrica e perda de massa.

As reduções foram realizadas seguindo-se os parâmetros relacionados na tabela a seguir:

Tabela 2. Parâmetros de redução utilizados nos experimentos

\begin{tabular}{|cc|}
\hline Fluxo de $\mathrm{H}_{2}\left(\mathrm{~cm}^{3} / \mathrm{min}\right)$ & $100 ; 200 ; 300 ; 400$ \\
\hline Tempo $(\mathrm{min})$ & 60 \\
\hline Temperatura $\left({ }^{\circ} \mathrm{C}\right)$ & 300 \\
\hline Pressão (Torr) & 4,0 \\
\hline Tensão DC $(\mathrm{V})$ & 540 \\
\hline
\end{tabular}

Inicialmente, foi produzido vácuo no reator para pressões menores do que 1 torr, e então os cadinhos foram aquecidos através de uma resistência elétrica inserida internamente no catodo. Quando a temperatura desejada era alcançada, a

* Contribuição técnica ao 69ำ Congresso Anual da ABM - Internacional e ao 14을 ENEMET Encontro Nacional de Estudantes de Engenharia Metalúrgica, de Materiais e de Minas, 21 a 25 de julho de 2014, São Paulo, SP, Brasil. 
resistência elétrica era desligada e a pressão do reator ajustada através da liberação do hidrogênio na vazão pré-estabelecida. Por fim, o plasma era ligado, dando início ao processo de redução.

Terminado o processo de redução as amostras foram resfriadas dentro do reator até a temperatura de $70^{\circ} \mathrm{C}$ para evitar uma possível reoxidação. Em seguida as amostras foram pesadas. A determinação da eficiência da redução foi realizada através do balanço de massa, difração de raio-x (DRX) e Microscopia eletrônica de Varredura (MEV).

\subsection{Difração de Raio-x (DRX)}

A evolução das fases formadas em função das diferentes condições de redução foram acompanhadas usando-se o equipamento de difração de raio-x da marca Bruker modelo D2 phaser instalado no laboratório de técnicas de análose instrumental do laboratório do IFES unidade Vitória.

\subsection{Microscopia Eletrônica de Varredura (MEV)}

Para acompanhar a evolução qualitativa da redução e verificar os aspectos morfológicos do óxido de cobre e do cobre metálico foram feitas imagens no MEV. As imagens foram realizadas utilizando o MEV microscópio eletrônico de varredura modelo EVO- MA10 da marca ZEISS pertencente ao laboratório do grupo de engenharia de superfícies do IFES unidade Vitória. A análise quantitativa da redução foi realizada através da análise de EDS acoplado ao MEV.

\section{RESULTADOS E DISCUSSÃO}

Após a redução foi realizado o balanço de massa. Considerou-se que a perda de massa durante a redução ocorre em função da perda de oxigênio que deixa o sistema na forma de vapor de água. Os percentuais de redução para os diferentes fluxos de $\mathrm{H}_{2}$ se encontram na tabela a seguir.

Tabela 2. Resultados calculados para a redução de $\mathrm{Cu}_{2}$

\begin{tabular}{|c|c|}
\hline $\begin{array}{c}\text { Fluxo de H2 } \\
(\mathrm{cm} 3 / \mathrm{min})\end{array}$ & Redução \% \\
\hline 100 & 50,82 \\
\hline 200 & 50,75 \\
\hline 300 & 44,86 \\
\hline 400 & 43,66 \\
\hline
\end{tabular}

As figuras de 2 a 5 foram obtidas através do ensaio de raio-x para a matéria-prima $\left(\mathrm{Cu}_{2} \mathrm{O}\right)$ e para as amostras de redução para os diferentes fluxos.

\footnotetext{
* Contribuição técnica ao 69 Congresso Anual da ABM - Internacional e ao 14을 ENEMET Encontro Nacional de Estudantes de Engenharia Metalúrgica, de Materiais e de Minas, 21 a 25 de julho de 2014, São Paulo, SP, Brasil.
} 


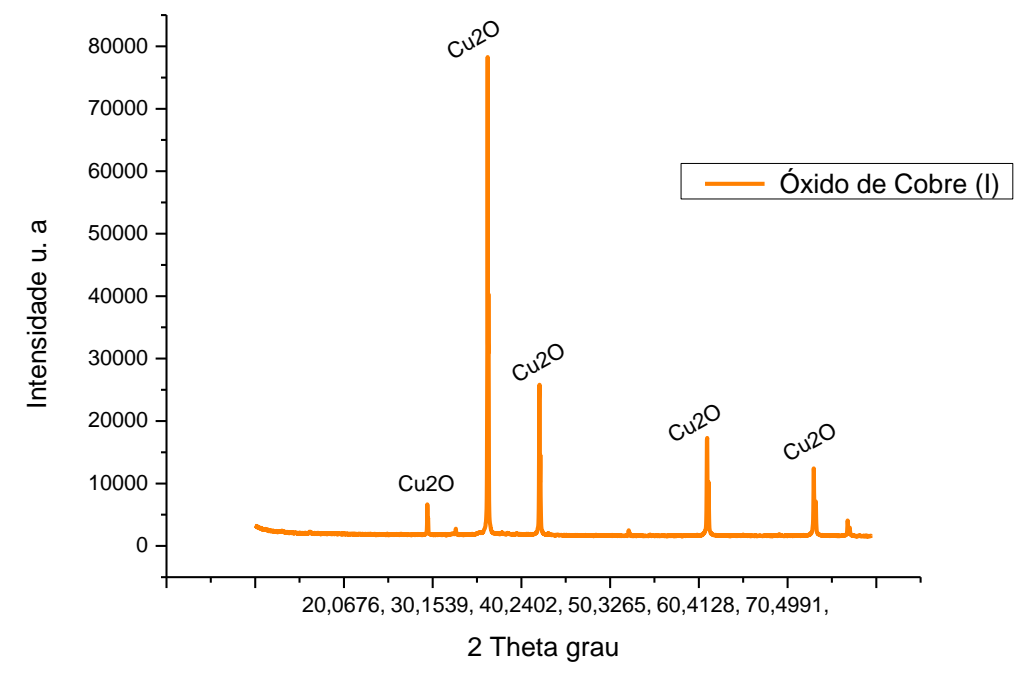

Figura 2: Espectro de difração de raio-x obtido para a amostra de $\mathrm{Cu}_{2} \mathrm{O}$ antes da redução.

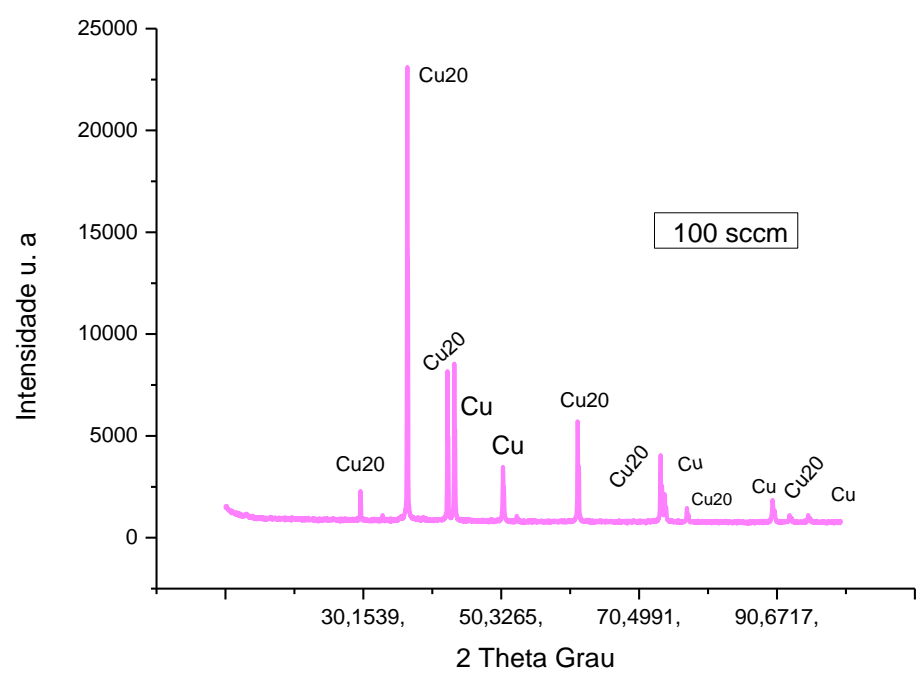

Figura 3: Espectro de difração de raio-x obtido para a amostra de reduzida a um fluxo de 100 $\mathrm{cm}^{3} / \mathrm{min}$ de $\mathrm{H}_{2}$.

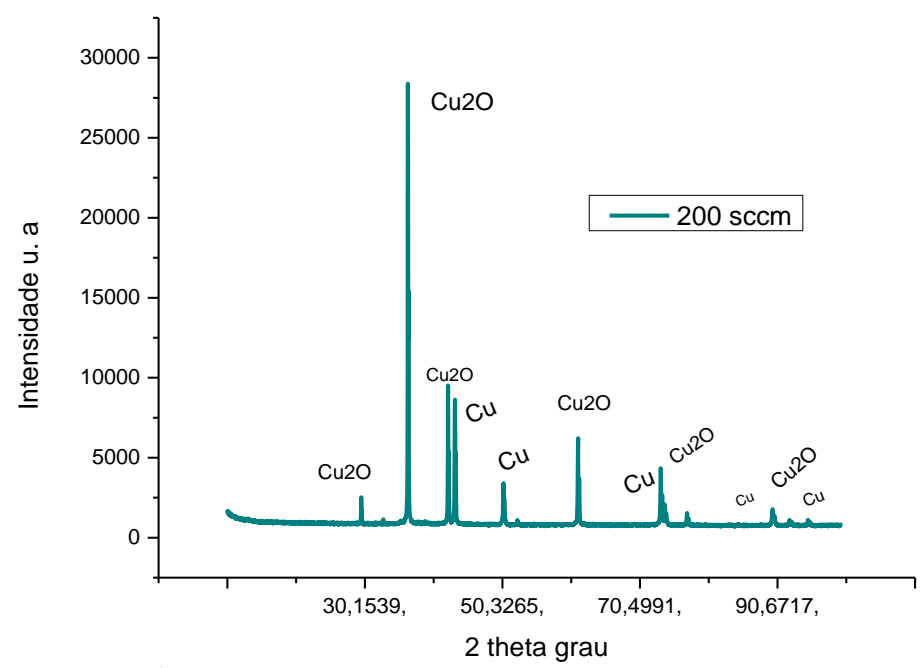

Figura 4: Espectro de difração de raio-x obtido para a amostra de reduzida a um fluxo de 200 $\mathrm{cm}^{3} / \mathrm{min}$ de $\mathrm{H}_{2}$.

* Contribuição técnica ao 69 Congresso Anual da ABM - Internacional e ao 14을 ENEMET Encontro Nacional de Estudantes de Engenharia Metalúrgica, de Materiais e de Minas, 21 a 25 de julho de 2014, São Paulo, SP, Brasil. 
A figura 8 mostra o resultado da análise de EDS realizada em pontos específicos da amostra reduzida com um fluxo de $200 \mathrm{~cm}^{3} / \mathrm{min}$ de $\mathrm{H}_{2}$.
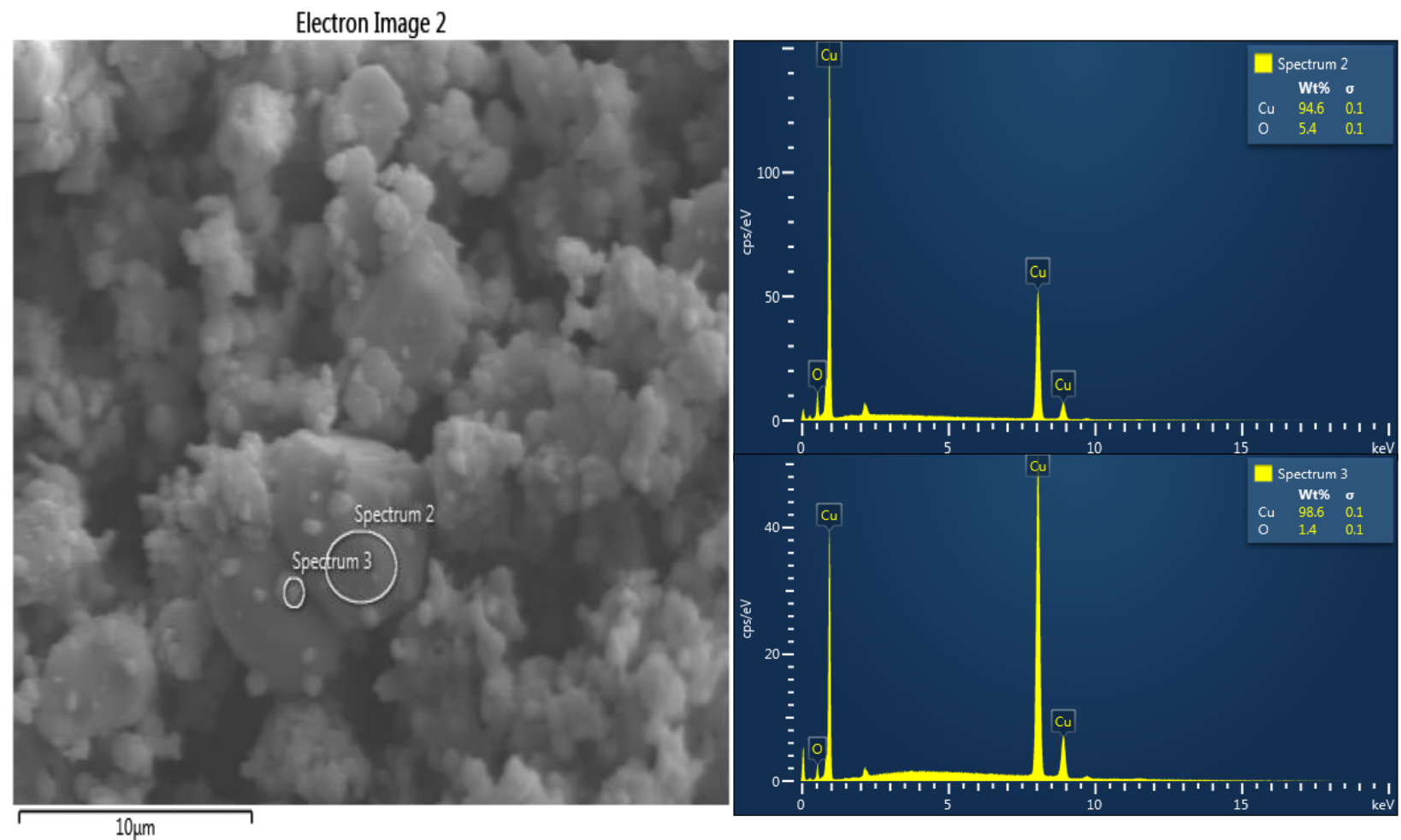

Figura 8: Resultado da composição química das duas regiões destacadas em (a).

Analisando os resultados descritos na tabela 2 observa-se que para os fluxos de $\mathrm{H}_{2}$ de 100 e $200 \mathrm{~cm}^{3} /$ min não houve uma diferença significativa no percentual de redução. Observa-se também que para valores maiores de fluxo (300 e 400 $\mathrm{cm}^{3} / \min$ ) o aumento da vazão do gás ocasionou uma queda na eficiência da redução. Estes resultados mostram que a influência do fluxo tende a apresentar um comportamento assintótico, no qual, acima de um dado valor ótimo um acréscimo no abastecimento do reagente gasoso tende a diminuir a redução.

Não existem muitos estudos na literatura sobre esta variável na redução a plasma. A maioria dos experimentos trabalha com valores de fluxo de $300 \mathrm{~cm}^{3} / \mathrm{min}$ e, conforme mostrado no presente trabalho, valores inferiores de $\mathrm{H}_{2}$ poderiam levar a reduções mais eficientes com economia de reagente [10].

Pode-se supor que um aumento no fluxo de gás não é acompanhado por um aumento na formação das partículas redutoras formadas no pelo plasma. Assim uma maior quantidade de $\mathrm{H}_{2}$ diminuiria a concentração dessas espécies no sistema e diminuiria a eficiência da obtenção do cobre metálico.

A figura 2 mostra o espectro da difração de raio-x da amostra de $\mathrm{Cu}_{2} \mathrm{O}$ antes da redução. Nela observa-se apenas a presença de picos relativos à presença desta fase, não havendo a presença de outros óxidos de cobre e nem de Cu metálico. Nas figuras 3 a 5 observa-se que para as reduções conduzidas a 100 e $200 \mathrm{~cm}^{3} / \mathrm{min}$ aparecem picos mais intensos da fase metálica do que os observados para as amostras reduzidas a um fluxo maior de $\mathrm{H}_{2}$.

Pela observação das figuras 6 e 7 é possível verificar a presença de pequenas partículas brilhantes na superfície de microaglomerados de óxido de cobre. Estas porções metálicas estão presentes em maior quantidade na figura 5 (redução a $100 \mathrm{~cm}^{3} / \mathrm{min}$ ) o que está de acordo com o resultado obtido pelo balanço de massa.

* Contribuição técnica ao 69ํㅡㄹ Congresso Anual da ABM - Internacional e ao 14ำ ENEMET Encontro Nacional de Estudantes de Engenharia Metalúrgica, de Materiais e de Minas, 21 a 25 de julho de 2014, São Paulo, SP, Brasil. 


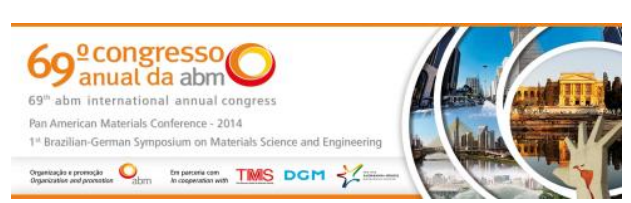

A figura 8 confirma através da espectroscopia de energia dispersiva (EDS) que as pequenas partículas claras observadas na superfície de partículas esféricas de óxido são de $\mathrm{Cu}$ metálico e que a redução é maior nas regiões mais aglomeradas (sinterizadas). Isto leva a crer que durante a redução há o coalescimento de algumas partículas através da fusão incipiente de algumas regiões. Novos estudos ainda devem ser realizados, mas os resultados obtidos fornecem importantes informações sobre o mecanismo e evolução da redução do óxido de cobre (I).

\section{CONCLUSÃO}

De acordo com os resultados obtidos nas análises de balanço de massa, DRX e MEV, pode-se concluir que:

- Através da utilização do plasma de hidrogênio a baixa temperatura é possível obter $\mathrm{Cu}$ metálico a partir de seu óxido estável $\left(\mathrm{Cu}_{2} \mathrm{O}\right)$, gerando como resíduo apenas vapor de água.

- O aumento do fluxo gasoso de $\mathrm{H}_{2}$ acima de $200 \mathrm{~cm}^{3 /} \mathrm{min}$ diminui a redução média de óxido de cobre (I).

- Para valores de fluxo entre 100 e $200 \mathrm{~cm} 3 / \mathrm{min}$ a contribuição de espécies oriundas do plasma é maior, aumentando assim o potencial redutor e a cinética do processo; $O$ conhecimento deste fato permite uma economia de $\mathrm{H}_{2}$ necessário ao processo.

- A fase metálica nucleia-se sobre a partícula de óxido e tende a coalescer e recobrir todo o $\mathrm{Cu}_{2} \mathrm{O}$.

Novos estudos estão sendo realizados para determinar a eficiência da redução pra valores de fluxo gasoso abaixo de $100 \mathrm{~cm}^{3} / \mathrm{min}$.

\section{Agradecimentos}

A FACITEC - Fundo de Apoio a Ciência e Tecnologia, da cidade de Vitória;

Ao IFES - Instituto Federal de Educação, Ciência e Tecnologia do Espírito Santo - Campus Vitória;

Ao PIBIC- Programa Institucional de bolsas de iniciação científica. IFES. Edital 2013/1

\section{REFERÊNCIAS}

1 Andrade MA, et al. Indústria do Cobre. BNDES- Bando Nacional de Desenvolvimento, 1997 p. 1-33. Disponível em WWW.bndes.org.br. Acesso em 20 Fev 2014.

2 Rubio JÁ. La metalurgia Del Cobre. AllM-, 1999. P.11-15. Disponível em < http://www.aim.es/publicaciones/bol2/13_Metalurgia_Cobre.pdf> Acesso em 20 jan 2014.

3 Ribeiro JAS. Balanço mineral Brasileiro 2001, DPNM-Departamento nacional de produção mineral, 2001. p.1-52. [ Acesso em 4 de dezembro de 2013]

4 Disponível em http://www.dnpm.gov.br/assets/galeriadocumento/balancomineral2001/cobre.pdp

5 Andrade MLA, de, et al. Indústria do Cobre. BNDES- Bando Nacional de Desenvolvimento, 1997 p. 1-33 [Acesso em 8 de dezembro de 2013]. Disponível em www.bndes.org.br.

\footnotetext{
* Contribuição técnica ao 69ำ Congresso Anual da ABM - Internacional e ao 14ํㅡㄹ ENEMET Encontro Nacional de Estudantes de Engenharia Metalúrgica, de Materiais e de Minas, 21 a 25 de julho de 2014, São Paulo, SP, Brasil.
} 
6 Mineração Caraíba S/A, 2009. Site institucional.[Acesso em 20 de janeiro de 2014] Disponível em http://www.minacaraiba.com/ .

7 Kim JY. Reduction of $\mathrm{CuO}$ and $\mathrm{Cu} 2 \mathrm{O}$ with H2: H Embedding and, JACS Articles, pp. 1-9, 13 Março 2003.

8 Bullard DB, Lynch DC. Reduction of titanium dioxide in a nonequilibrium hydrogen plasma. Metallurgical and Materials Transaction B, 1997; 28B: 1069-1080.

9 Figueroa CA. Propriedades microscópicas e macroscópicas de superfície em ligas ferrosas implantadas com nitrogênio. Tese de Doutorado. Instituto de Física "Gleb Wataghin". Campinas, SP, 2004.

10 Zangh Y, Ding W, LU Z. et al. Reduction oh TiO2 with hydrogen cold plasm in dc pulsed glow discharge. Trans. Nonferrous met. soc. China, 2005; 15.

* Contribuição técnica ao 69ำ Congresso Anual da ABM - Internacional e ao 14을 ENEMET Encontro Nacional de Estudantes de Engenharia Metalúrgica, de Materiais e de Minas, 21 a 25 de julho de 2014, São Paulo, SP, Brasil. 